自动化技术

\title{
带电感性直流賀載扼流磁放大器的 静、动态过程理論分析
}

\author{
墦 守魯
}

磁放大器的应用, 现在已经非常广泛. 性现象（磁芯磁化曲线和二极管特性）的元 磁放大器工作的靜、动态物理过程的分析, 件, 其输入和输出波形畸变得很厉害, 从而 自然地引起人们的很大重视. 因为只有正确 给磁放大器的特性分析, 特別是带非电阻性 地理解了有关磁放大器的靜、动态特性, 才 负载的磁放大器的动特性分析, 带来很大困 有可能进行合理的系统设计和分析.

但是, 磁放大器是一个典型的利用非线 难. 有关电感性淔流负载的扼流磁放大器的 分析, 便是一例.

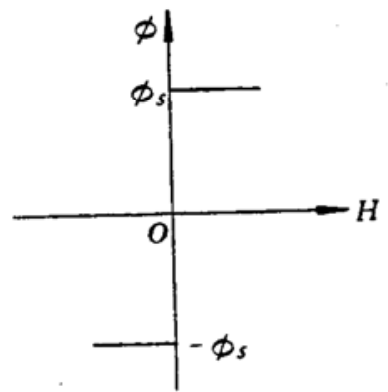

(a)

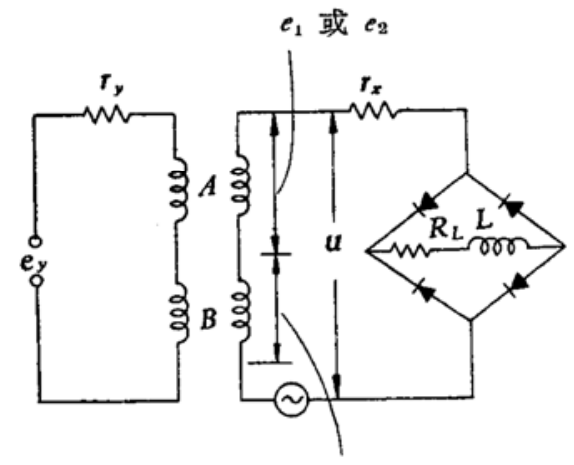

$e_{2}$ 或 $e_{1}$

$e_{\sim}=E_{m} \sin \omega t$

(b)

图 1 （a）磁芯 $A$ 和 $B$ 的磁化曲线；（b）带电感性直流负载的扼流磁放大器线路图

带电感性直流负载的扼流磁放大器, 是 磁放大器最基本的典型线路之一，其线路看 来很简单，但其磁电耦合关系和靜、动态物 理过程，却是其他带电感性直流负载的磁放 大器线路中最复杂的.

近十余年来，先后发表过不少有关这一 专题的论交 ${ }^{[1-10]}$. 但是, 必须指出, 这些论
交迄今劣未完全解决它们所提出的问题. 它 们或者是在理论分析中一些主要假设的概念 上就存在原则性的问题 ${ }^{[-3,5-7]}$, 或者只是从 线性等效线路等概念来推导得一些很近似的 公式 ${ }^{[3,4]}$ ，而沒有触及本问题的 实质一磁 放大器的各主要参变量在整个磁化过程中的 动态关系. 因此, 实验表明, 它们的理论分析 


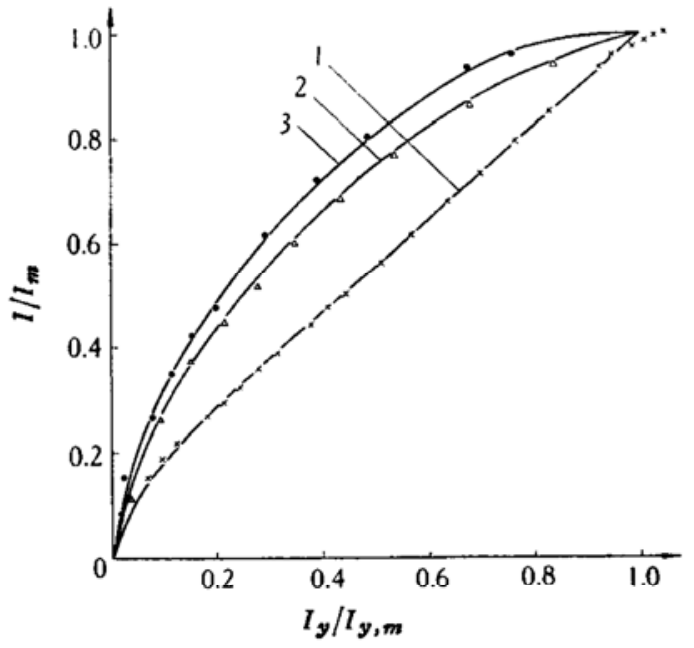

图 2 在不同的电路阻值比 $\left(r_{x} / r_{y}\right.$ 和 $\left.R_{L} / r_{y}\right)$ 情况下，带电感性直流负载扼流磁放大器的法 静态特性曲线（图中实线系本文理论计算曲 线，小圆点、三角和十字则分別系各曲线的实 测数据）

曲线 1. $R_{L} / r_{y}=0.081, r_{x} / r_{y}=0.0041$,

$$
\begin{aligned}
& R_{L}=295 \Omega, L=31.7 h, \\
& r_{x}=15 \Omega, r_{y}=3650 \Omega ;
\end{aligned}
$$

曲线 2. $R_{L} / r_{y}=10, r_{x} / r_{y}=0.1$, $R_{L}=1535 \Omega, L=31.7 \mathrm{~h}$, $r_{x}=16.7 \Omega, r_{y}=148 \Omega$

曲线 3. $R_{L} / r_{y}=2.8, r_{x} / r_{y}=2.9$, $R_{L}=365.4 \Omega, L=31.7 \mathrm{~h}$, $r_{x}=381.9 \&, r_{y}=131 \Omega$

结果只适用于很局限的特例而已.

本交对照实测的电流波形变化，严格地 遵循实际线路中存在的物理关系, 推导出各 电流和电压的半波平均值表达式，进而推证 得带电感性直流负载扼流磁放大器中各参 变量之间存在的正确的靜、动态函数关系及 其传递函数公式; 具体地指出了上述各交 献 ${ }^{[1-7,9]}$ 中那些有爭论之点与严格的表达式和 结论之间究竟有多大的分歧; 最后, 我对上 述各文献和本文的理论分析结果进行实验验 证和比较.

按过去各交献的理论分析 ${ }^{[1-3,5,6,10]}$, 带电 感性直流负载扼流磁放大器的法靜态特性, 在所假设条件下，系只与饱和角有关，而与 线路的其他参变数无关 (其法特性如图 2 中 曲线 2). 至于动态传递函数, 则有的交 献 ${ }^{[1-4,7]}$ 论证其形式为分母二阶和分子一阶, 而有的 ${ }^{[5]}$ 则论证为分母二阶和分子二阶.

本文的分析证明: 在 $\omega T_{H} \gg 1$ 且 $\beta \gg \theta$ 的假设条件下，电感性直流负载扼流磁放大 器的靜态特性不仅与饱和角有关, 且与阻値 比 $\left(r_{x} / r_{y}\right.$ 和 $R_{L} / r_{y}$ ) 密切相关 (如图 2 中曲线 $1 、 2$ 和 3$)$, 其公式为

$$
\begin{gathered}
I_{y} / I_{y, m}=\left(\frac{1+\cos \beta}{2}\right) \cdot\left(\frac{\frac{r_{x}}{r_{y}}+\frac{R_{L}}{r_{y}}}{\frac{\beta}{\pi}+\frac{r_{x}}{r_{y}}+\frac{R_{L}}{r_{y}} K_{i s}}\right) \\
I / I_{y} \triangleq K_{i s}=\pi /(\pi-\beta)
\end{gathered}
$$

和

$$
K_{i \delta} \triangleq \frac{\Delta I}{\Delta I_{y}}=K_{i s} \frac{1}{1+\frac{1+\cos \beta}{(\pi-\beta) \sin \beta}\left[\frac{1}{1+\frac{K_{i s}}{\sin \beta}\left(1-\frac{2 \beta}{\pi}-\frac{r_{x}}{r_{y}}\right) \frac{E_{y}}{E_{m}}}\right]}
$$

问题的公式.

文中引述的大量实验证明: 本交公式 一阶, 郎,

$$
\mathrm{Y}(s)=K_{i \delta}\left(1+T_{a} s\right) /\left(1+T_{1} s+T_{2}^{2} s^{2}\right)
$$

这些结果，大不同于现有交献中对同一 (1)、(2) 和 (3)，是正确的，是带电感性直流 负载扼流磁放大器的靜态特性的通解. 它们 
表 1 图 3 中各文(包括本文)理论计算结果比较

\begin{tabular}{|c|c|c|c|}
\hline 根据 & $\begin{array}{l}\text { 起始电流 } \\
I_{0}(m \alpha)\end{array}$ & $\begin{array}{l}\text { 稳态电流 } \\
I_{\infty}(m \alpha)\end{array}$ & 传递函数 $\left(Y(s)=\frac{\Delta I(s)}{\Delta I_{\boldsymbol{y}}(s)}\right)$ \\
\hline 实 测 伹 & 7.24 & 19.9 & 实拍示波器图 (图 3a) \\
\hline 交献 $[1 、 4]$ & 10.8 & 43.5 & $32.7 \frac{1}{1+15.7 \times 10^{-5} s+1.93 \times 10^{-5} s^{2}}$ \\
\hline 交 献 [2] & 10.8 & 50 & $50 \frac{1}{1+24 \times 10^{-5} s+2.95 \times 10^{-5} s^{2}}$ \\
\hline 交 献 [3] & 10.8 & 43.5 & $32.7 \frac{1}{1+0.1034 s+1.93 \times 10^{-5} s^{2}}$ \\
\hline 文 献 [5] & 10.8 & 43.5 & $32.7 \frac{1+1.7 \times 10^{-3} s+0.582 \times 10^{-6} s^{2}}{1+45.2 \times 10^{-3} s+2.65 \times 10^{-5} s^{2}}$ \\
\hline 公式 (4) & 7.1 & $26.1^{*}$ & $19 \frac{1+0.625 \times 10^{-3} s}{1+14.9 \times 10^{-3} s+3.09 \times 10^{-5} s^{2}}$ \\
\hline
\end{tabular}

* 在此实验中, 输入控制信号增量故意选择很大 $\left(\Delta I_{y} / I_{y}, m \cong 20 \%\right)$ 以夸大靜特性非线性的影响, 因此根 据式(4)计算的 $I_{\infty}$ 与实测值相差达 $37 \%$, 远较正常情兄下为大.

正确地反映出有关磁放大器的一些参变量和 靜态特性之间存在的函数关系. 而目前一般

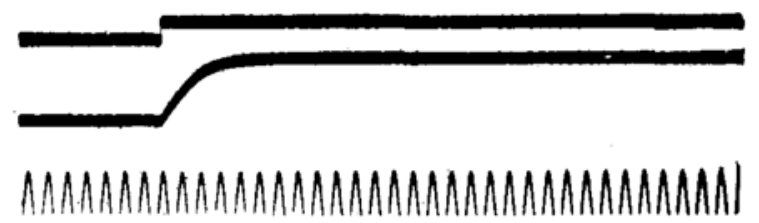

(a)

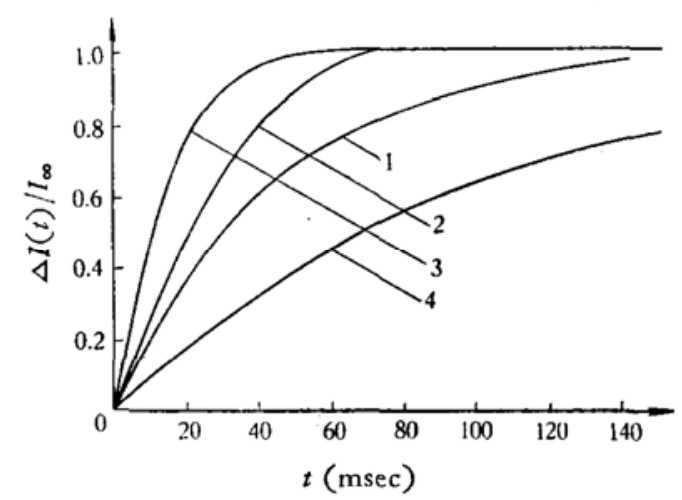

(b)

图 3 (a) 在 $R_{L} / r_{y}=0.0975$ 和 $r_{x} / r_{y}=0.0062$ 情况下, 堵量输入反应的示波器实拍曲线; (b) 各文理论计算曲线与实测曲线比较*

曲线 1. 文献 [5]; 曲线 2. 实测曲线; 曲线 3. 本交理论曲线; 曲线 4. 交献[3]

* 交献 $[1,2,3]$ 的理论曲线在本图中未画出, 因它们 与实测曲线相差太大 (表 1).
通用的靜态公式则只是本交公式的一个特 解. 此外，本交推导出的、描述动态反应的 传递函数公式(4)也证明是正确的 (见图 3、 表1); 在推理和物理概念上，它不存在以往 各交献存在的那些问题，且与实验结果亦符 合得最好. 一些过去所难以解释的“不正常” 现象，现在已可以根据本交推导得的这些公 式, 给以确切的解释.

\section{数 学 等 号}

I 负载电流(牛波平均值, 下同)

$I_{y}$ 折合到交流绕组边的等效控制电流

$I_{y, m}$ 折合到交流绕组边的等效最大控制电流

$K_{i}$ s 稱态电流堦盛

$K_{i \delta}$ 电流增量增谷

$\beta$ 饱和角

$r_{x}$ 交流绕组电阻 (包括交流电源內阻)

$r_{y}$ 折合到交流绕组边的等效控制迴路电阻

$R_{L}$ 和 $L$ 负载电阻（包括整流电桥二极管导向 电阻)和电感(本文中假设 $\omega L / R_{L} \gg 1$ )

$E_{\boldsymbol{y}}$ 折合到交流绕组边的控制电压

$E_{m}$ 交流激励电压最大值 
$T_{a} 、 T_{1}$ 和 $T_{2}$ 动态反应时间常数, 其值可分別表 示为线路中各参变量的函数(详见正文)

\section{参考 文 献}

[1] Storm, H. F., Magnetic Amplifiers, New York, John Wiley \& Sons, Inc., pp. 170-192, 1955.

[2] Розинблат М. А., Магнитныи усилители, Треье Изд., Москва: Издательство советское радио, стр. 268-277, 1960.

[3] Ху Ц. Я. и Щубенко В. А., Электричество, № $10,35-40$ (1960).
[4] Сафрис Л. В., Авто. и телемех., 14 [3], 228238 (1958).

[5] Льов Е. Л., ibid., 22 [12], 1956-1972 (1961).

[6] Storm, H. F., Trans. A.I.E.E., 71, Pt. 1, 335343 (1952).

[7] Storm, H. F., ibid., 72, Pt. 1, 182-192 (1953).

[8] Finizi, L. A. \& Jackson, R. R., Trans. A. I. E. E., 73, Pt. 1, 270-288 (1954).

[9] 胡家跃, 自动化学报, 2[2], 97-107 (1964).

[10] Васильева Н. П., Авто. и телемех., №6, $544-553$ (1954). 